\title{
Study of Fatty Acids Profile in Kernel Oil of Heynea trijuga Roxb. Ex Sims. (syn. Trichilia connaroides (Wight \& Arn.) Bentv.)
}

\author{
Ashutosh K. Mittal and Shishir Tandon \\ Department of Chemistry, Division of Agricultural Chemicals, College of Basic Sciences and Humanities, \\ G. B. Pant University of Agriculture and Technology, Pantnagar, Uttarakhand 263 145, India
}

Correspondence should be addressed to Shishir Tandon; shishir_tandon2000@yahoo.co.in

Received 28 January 2016; Revised 30 June 2016; Accepted 10 July 2016; Published 17 January 2017

Academic Editor: Artur M. S. Silva

Copyright (C) 2017 Ashutosh K. Mittal and Shishir Tandon. This is an open access article distributed under the Creative Commons Attribution License, which permits unrestricted use, distribution, and reproduction in any medium, provided the original work is properly cited.

Seed oils have been used for centuries by communities as food, medicine, cosmetic applications, and fuel. Recently, there has been a renewed interest in these nontimber forest products specifically for use in cosmetic formulations. The fatty acid compositions of kernel oil of Heynea trijuga was analyzed by GC-FID. The results showed that the oil content was 37.61 percent (w/w) in seed. Seed oil was rich in unsaturated fatty acids. Important fatty acids present were palmitic acid (22.12\%), stearic acid (7.51\%), oleic acid (25.20\%), and linoleic acid (11.65\%).

\section{Introduction}

The multibillion dollar natural products industry (food, beverages, cosmetics, herbal medicines, and pharmaceuticals) has grown enormously with an annual growth rate of 15$20 \%$ [1]. In 2005, it was valued at US\$65 billion/year with a US $\$ 1$ billion/year growth in the global sales of natural and organic cosmetics specifically for the wellness industry [2]. Oils extracted from plant sources have a rich history of use by local people as a source of food, energy, and medicine and for cosmetic applications. It has been used in the production of lubricants, soaps, and personal care products, as well as in the topical treatment of various conditions such as hair dandruff muscle spasms, varicose veins, and wounds $[3,4]$. In recent years, demand for seed oils as ingredients for food, cosmetics, and biofuel has greatly increased as industry seeks natural alternatives. The global production of seed oils increased exponentially, creating pressure on countries providing the raw material to meet the growing demand. Currently, the global supply is obtained from only about 15 plant species out of nearly half a million known to man, highlighting the greater potential for food, cosmetics, and biofuel [5].
Heynea trijuga Roxb. ex Sims. (syn. Trichilia connaroides (Wight \& Arn.) Bentv.) (family: Meliaceae) plant is found in Indian subcontinent [6]. It is distributed from sub-Himalayan tract of Kumaon, Sikkim, Khasi Hills, and Manipur to Eastern and Western Ghats. Apart from India, it is also distributed in Burma, Tonkin, Cambodia, Malay Peninsula, and Sumatra [7]. The plant possesses many medicinal values; the bark and leaves possess bitter and tonic properties and a decoc tion of the leaves is taken for cholera [8]. The roots of T. connaroides are used as a Chinese drug to treat arthritis, pharyngitis, tonsillitis, and other ailments [9]. The main chemical constituents of T. connaroides are the bitter principles triterpenoids or limonoids [10]. The chemical properties of this plant of Indian origin are not fully known and are under investigation by workers [11]. An excellent review work on phytochemistry and ethnopharmacology has been done by Garg [12]. Compounds belonging to this group exhibit a range of pharmacological/biological activities including insecticidal, antifeedant, and growth regulating activities as well as antimicrobial, antiviral, antimalarial, analgesic and anti-inflammatory, anthelmintic, and anticancerous activities on humans [12-15]. 
The information on Indian seed oils is scattered in literature. As per our literature search, there is very little study on seed fatty acid profile of this plant; thus, present study was aimed at identification and characterization of chemical composition of fatty acids profile of seed oil of $H$. trijuga growing in Kumaon hills.

\section{Materials and Methods}

2.1. Chemicals Used. GC grade n-hexane, petroleum ether, and sodium sulfate AR grade were procured from E. Merck (India) Limited, Mumbai. Identification of fatty acids was based on the standard mixture of fatty acids methyl esters obtained from Sigma-Aldrich Chemicals, USA.

2.2. Plant Collection and Identification. Plant material, that is, red matured fruits were collected in the month of October to November from Nainital district of Kumaon region. Plants (Voucher number M-29) were identified by Professor Y. P. S. Pangtey, Taxonomist, Department of Botany, Kumaon University, Nainital, and reconfirmed at Forest Research Institute (FRI), Dehradun (herbarium number M29). The herbarium is maintained at Department of Chemistry (Division Agricultural Chemicals), CBS \& H, G. B. Pant University of Agriculture and Technology, Pantnagar (India).

2.3. Preparation of Seed Oil. The material (matured fruit) was shade dried and seeds were separated from seed coat/pericarp. Kernel oil was obtained by refluxing seeds for 12 hours with petroleum ether in Soxhlet apparatus till complete extraction of oil was done. The petroleum ether was evaporated in rotatory vacuum evaporator at $35 \pm 10^{\circ} \mathrm{C}$ leaving behind seed oil.

The kernel oil was esterified into methyl esters by sodium methoxide catalyzed transesterification as given by Cristie [16]. The mixture was refluxed for 6-8 hours on water bath. The methyl esters were extracted in n-hexane, washed with hot water, and dried over anhydrous sodium sulfate [1720].

2.4. GC Condition. Fatty acid methyl esters were analyzed using a Hewlett-Packard GC 5890 series II, equipped with HP-225 (50\% cyanopropyl; 50\% phenylmethyl polysiloxane) capillary column $(30 \mathrm{~m} \times 0.25 \mathrm{~mm}$ id $\times 0.25 \mu \mathrm{m})$ and flame ionization detector (FID), injection port temperature was set at $250^{\circ} \mathrm{C}$, and detector temperature was adjusted at $250^{\circ} \mathrm{C}$. The temperature of column was programmed with linear increase from $50^{\circ} \mathrm{C}$ to $220^{\circ} \mathrm{C}$ with a rate of $4^{\circ} \mathrm{C} / \mathrm{min}$ and was held for 30 minutes at $220^{\circ} \mathrm{C}$. The flow rate of carrier gas was $1 \mathrm{~mL} / \mathrm{min}$; sample injected was $1 \mu \mathrm{L}$ with split ratio of $50: 1$.

For qualitative estimation of fatty acids, the retention time of standard fatty acid methyl esters was compared with the retention time of the peaks obtained in the sample. Standard solution of fatty acid methyl ester was prepared by dissolving $1 \mu \mathrm{L}$ of fatty acid mixture solution in $1 \mathrm{~mL}$ GC grade $\mathrm{n}$-hexane.
TABle 1: Percentage of oil and fatty acids composition of Heynea trijuga seed kernel oil.

\begin{tabular}{lc}
\hline Total oil (\%) on dry weight basis & $37.61 \pm 0.2$ \\
Saturated fatty acids & Percentage \\
Butyric acid (4:0) & $1.59 \pm 0.01$ \\
Undecanoic acid (11:0) & $0.23 \pm 0.01$ \\
Lauric acid (12:0) & $2.50 \pm 0.01$ \\
Myristic acid (14:0) & $2.64 \pm 0.02$ \\
Pentadecanoic acid (15:0) & $0.45 \pm 0.01$ \\
Palmitic acid (16:0) & $22.12 \pm 0.04$ \\
Heptadecanoic acid (17:0) & $4.67 \pm 0.01$ \\
Stearic acid (18:0) & $7.51 \pm 0.02$ \\
Arachidic acid (20:0) & $0.80 \pm 0.01$ \\
Heneicosanoic acid (21:0) & $1.07 \pm 0.01$ \\
\hline Total saturated fatty acids & 43.58 \\
\hline Unsaturated fatty acids & \\
Mono unsaturated fatty acids & $1.02 \pm 0.02$ \\
Palmitoleic acid (16:1) & $25.20 \pm 0.05$ \\
Oleic acid (18:1) n9c & $1.24 \pm 0.01$ \\
Elaidic acid (18:1) n9t & $5.53 \pm 0.01$ \\
Cis-11-Eicosenoic acid (20:1) n9 & $2.59 \pm 0.01$ \\
Erucic acid (20:1) n9 & $3.23 \pm 0.01$ \\
Nervonic acid (24:1) n9 & \\
Polyunsaturated fatty acids & $0.70 \pm 0.01$ \\
Linoleic acid (18:2) n6c & $3.19 \pm 0.01$ \\
$\quad$-Linolenic acid (18:3) n6 & $0.40 \pm 0.02$ \\
$\alpha$-Linolenic acid (18:3) n3 & $0.02 \pm 0.01$ \\
Arachidonic acid (C20:4) n6 & \\
Cis-11,14-Eicosadienoic acid (C20:2) n6 & \\
Cis-4,7,10,13,16,19-Docosahexaenoic acid & \\
(C22:6) n3 & \\
\hline Total unsaturated fatty acids & \\
\hline
\end{tabular}

\section{Results and Discussion}

Total oil content in kernel was 37.61 percent ( $\mathrm{g} / 100 \mathrm{~g}$ dry wt kernel) of which $43.58 \%$ constitutes of saturated fatty acids and $54.81 \%$ of unsaturated fatty acids. Total fatty acids identified were twenty-two constituting $98.39 \%$ of the total oil percentage. The seed oil was rich in unsaturated fatty acids compared to saturated fatty acids. Monounsaturated fatty acids (MUFA) were dominating (38.81\%) when compared to polyunsaturated fatty acids (PUFA) (16.0\%). Important unsaturated and saturated fatty acids present in high percentages were palmitic acid (22.12\%), stearic acid (7.51\%), oleic acid (25.20\%), and linoleic acid (11.65\%) (Table 1). Unidentified fatty acids constitute only $3.47 \%$.

The kernel oil of $H$. trijuga collected from Kumaon region (India) has different oil composition as shown by other workers. Seeds of $H$. trijuga contains $34.7 \%$ oil, and the composition of important fatty acid was a follows: palmitic acid $(8.5 \%)$, stearic acid (4.8\%), oleic acid (81.8\%), linoleic acid (2\%), and linolenic acid (1.3\%) [21]; another analysis on same 
plant seed oil indicates presence of $33.1 \%$ oil content with oleic acid $(62.8 \%)$ and linoleic acid (21.3\%) as the major constituent [22]. The variation in oil composition of different types may be due to different species/varieties, agroclimatic conditions, ecological conditions, or pedology. Linoleic acid is the most frequently used fatty acid in cosmetic products as it is antiinflammatory, moisturises the skin, and aids in the healing process of demitasses, sunburns, and acne [23]. Oleic acid is also reported to be an effective percutaneous absorption enhancer [21]. Skin permeation enhancement effects were also recorded for linoleic, lauric, myristic, and stearic acids [23]. Presence of these acids in good percentage potentiates the wide use of $T$. connaroides seed oil for cosmetic/beauty purposes.

\section{Competing Interests}

The authors declare that they have no competing interests.

\section{References}

[1] I. Vermaak, G. P. P. Kamatou, B. Komane-Mofokeng, A. M. Viljoen, and K. Beckett, "African seed oils of commercial importance-cosmetic applications," South African Journal of Botany, vol. 77, no. 4, pp. 920-933, 2011.

[2] N. Nemarundwe, G. Le Breton, and I. Chibaya, "Linking rural producers of natural products to global markets for poverty alleviation in southern Africa," in Proceedings of the 13th World Forestry Congress, Buenos Aires, Argentina, October 2009, http://www.cfm2009.org/es/programapost/trabajos/ Linking_producers_FD.pdf.

[3] N. Zimba, S. Wren, and A. Stucki, “Three major tree nut oils of southern central Africa: their uses and future as commercial base oils," International Journal of Aromatherapy, vol. 15, no. 4, pp. 177-182, 2005.

[4] E. Chivandi, B. C. Davidson, and K. H. Erlwanger, "A comparison of the lipid and fatty acid profiles from the kernels of the fruit (nuts) of Ximenia caffra and Ricinodendron rautanenii from Zimbabwe," Industrial Crops and Products, vol. 27, no. 1, pp. 29-32, 2008.

[5] Y. C. Mitei, J. C. Ngila, S. O. Yeboah, L. Wessjohann, and J. Schmidt, "NMR, GC-MS and ESI-FTICR-MS profiling of fatty acids and triacylglycerols in some Botswana seed oils," Journal of the American Oil Chemists' Society, vol. 85, no. 11, pp. 10211032, 2008.

[6] The Plant List, Heynea trijuga Roxb. ex Sims, 2010, http:// www.theplantlist.org/tpl/record/kew-2849031.

[7] K. R. Kirtikar and B. D. Basu, Indian Medicinal Plant, vol. 1, Periodical Experts Book Agency, New Delhi, India, 1993.

[8] The Wealth of India, A Dictionary of Indian Raw Materials, vol. 5, Council of Scientific and Industrial Research, New Delhi, India, 1997.

[9] Z.-L. Geng, X. Fang, Y.-T. Di, Q. Zhang, Y.-M. Shen, and X.-J. Hao, "A new limonoid from Trichilia connaroides," Zeitschrift für Naturforschung B, vol. 65, no. 6, pp. 1-3, 2010.

[10] A. Roy and S. Saraf, "Limonoids: overview of significant bioactive triterpenes distributed in plants kingdom," Biological and Pharmaceutical Bulletin, vol. 29, no. 2, pp. 191-201, 2006.

[11] N. Senthilkumar, S. Murugesan, and K. B. Vijayalakshmi, "GCMS-MS analysis of Trichilia connaroides (Wight \& Arn.) Bentv
(Meliaceae): a tree of ethnobotanical records," Asian Journal of Plant Science and Research, vol. 2, no. 2, pp. 193-197, 2012.

[12] G. Garg, "Trichilia connaroides wight and arnott: ethnobotany, phytochemistry and pharmacology," Chinese Journal of Natural Medicines, vol. 9, no. 4, pp. 241-248, 2011.

[13] S. Tandon, A. K. Mittal, and A. K. Pant, "Growth-regulatory activity of Trichilia connaroides (syn. Heynea trijuga) leaf extracts against the Bihar hairy caterpillar Spilosoma obliqua (Lepidoptera: Arctiidae)," International Journal of Tropical Insect Science, vol. 29, no. 4, pp. 180-184, 2009.

[14] G. S. Prasanna and P. Ashok, "Antihyperhomocysteinemic and antihyperlipidemic effect of Trichilia connaroides in methionine-induced hyperhomocysteinemic animals," Indian Journal of Pharmacology, vol. 43, no. 2, pp. 203-206, 2011.

[15] R. Kumar, G. Verma, O. Prakash, and A. K. Pant, "Head space GC/MS analysis of volatile constituents of Trichilea connaroides wight and Arn. Extracts and their in vitro anti-plasmodium activity against Plasmodium falciparum isolates," Research Journal of Phytochemistry, vol. 5, no. 1, pp. 41-47, 2011.

[16] W. W. Cristie, "Fatty acid derivatives," in Gas Chromatography and Lipids, W. W. Cristie, Ed., pp. 64-84, Bell and Bain, Glasgow, UK, 1989.

[17] S. Tandon and R. C. Sawhney, "Fatty acid composition of seed oil of seabuckthorn collected from Leh region," The Global Seabuckthorn Research and Development, vol. 5, no. 2, pp. 4147, 2007.

[18] S. Tandon, P. Jayamurthy, and R. C. Sawhney, "Fatty acid composition of leaf oil of seabuckthorn collected from Leh region," The Global Seabuckthorn Research and Development, vol. 1, no. 7, pp. 41-43, 2009.

[19] S. Tandon and R. C. Sawhney, "Fatty acid composition of fruit pulp of seabuckthorn collected from Leh region," The Global Seabuckthorn Research and Development, vol. 4, no. 3, pp. 1824, 2005.

[20] V. Singh, R. K. Gupta, S. Tandon, and R. C. Sawhney, "Fatty acid composition of fruit pulp and seed oils of some high yielding forms of Seabuckthorn in Indian Himalayas," in Seabuckthorn (Hippophae L.): A Multipurpose Wonder Plant, V. Singh, Ed., vol. 3 of Advances in Research and Development, pp. 215-222, Daya Publishing House, New Delhi, India, 2008.

[21] K. Sundar Rao and G. Lakshminarayana, "Characteristics and composition of six newer seeds and the oils," Lipid/Fett, vol. 89, no. 8, pp. 324-326, 1987.

[22] R. Dayal, M. Sharma, and R. Sharma, "Fatty acid composition of Heteropanax fragrans and Heynea trijuga seeds," Journal of Lipid Science \& Technology, vol. 39, no. 2, pp. 61-62, 2007.

[23] A. Mittal, U. V. S. Sara, A. Ali, and M. Aqil, "Status of fatty acids as skin penetration enhancers-a review," Current Drug Delivery, vol. 6, no. 3, pp. 274-279, 2009. 

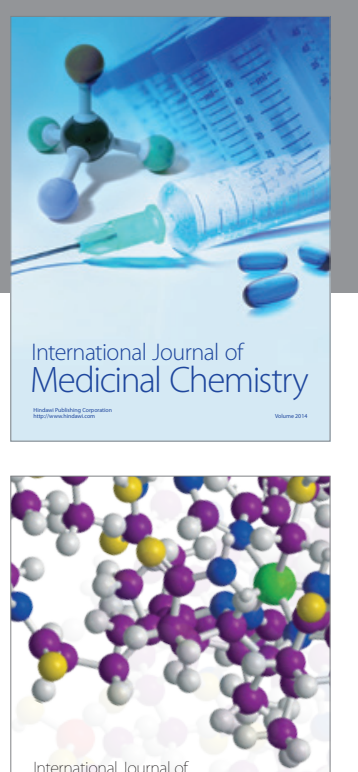

Carbohydrate Chemistry

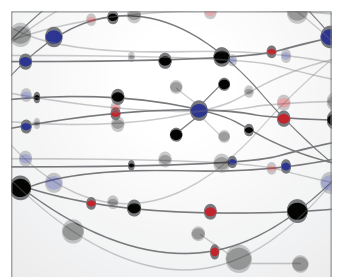

The Scientific World Journal
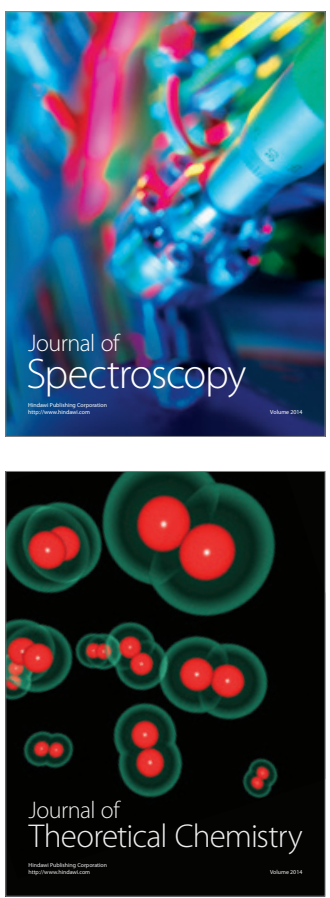
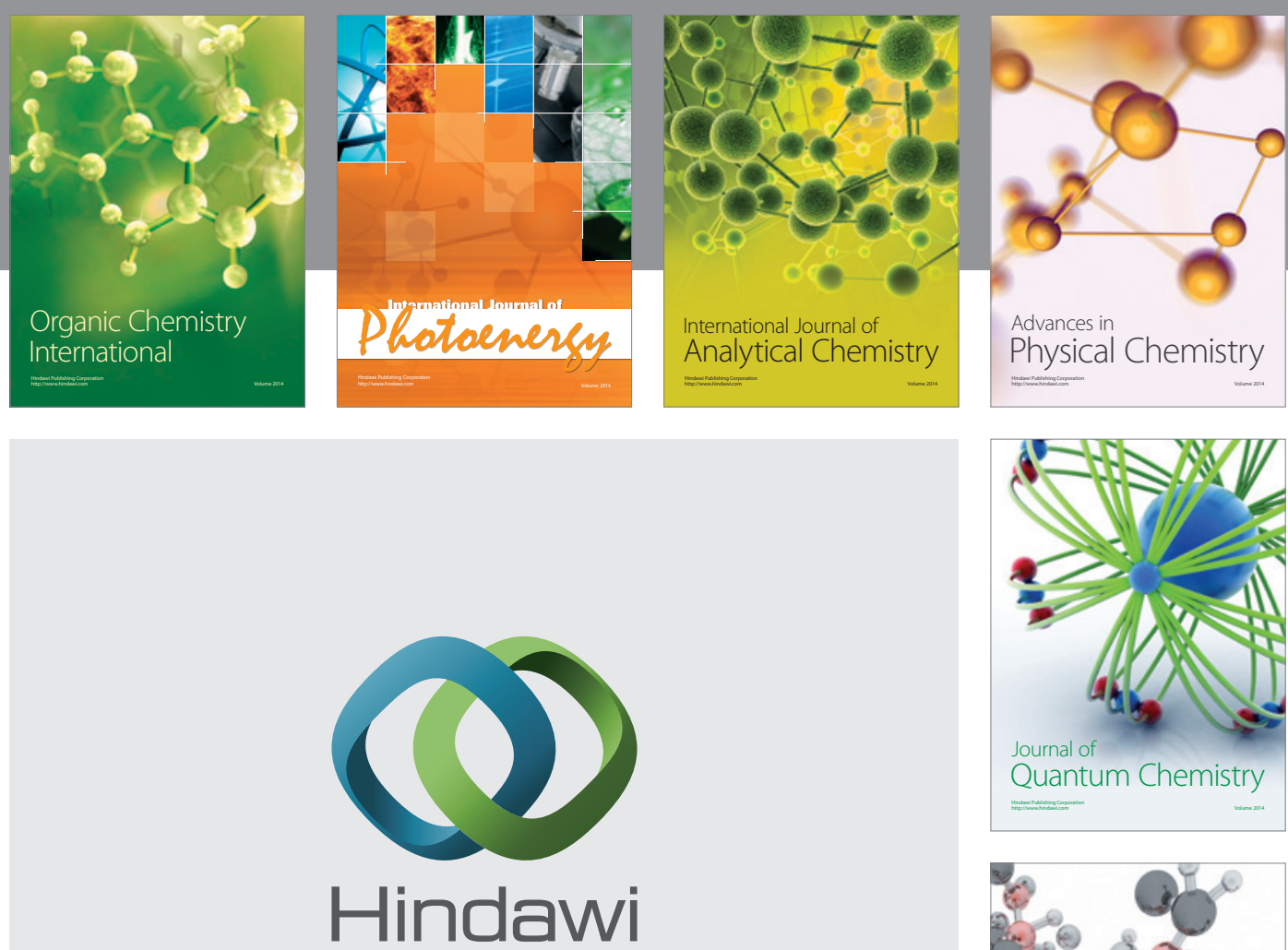

Submit your manuscripts at

https://www.hindawi.com

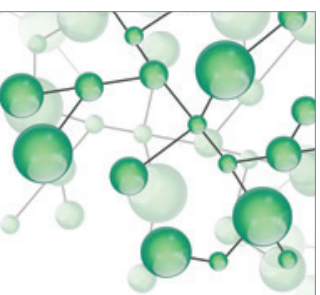

International Journal of

Inorganic Chemistry
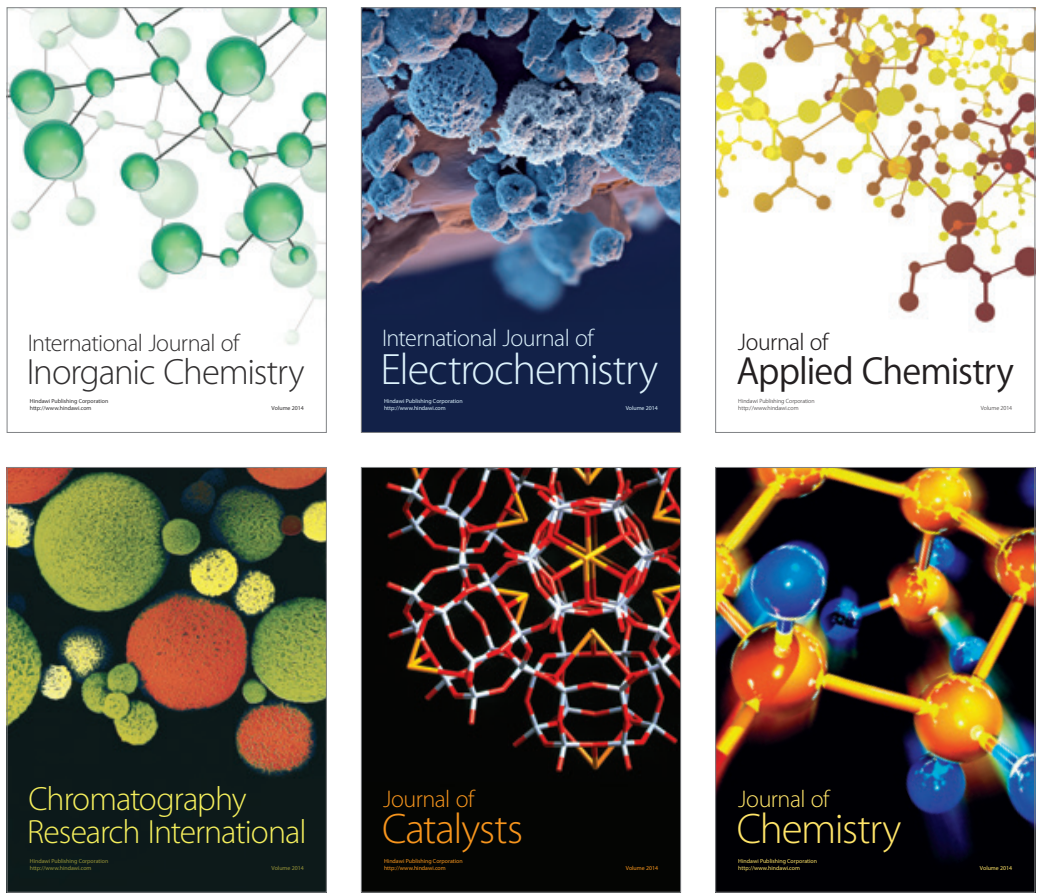

Journal of

Applied Chemistry
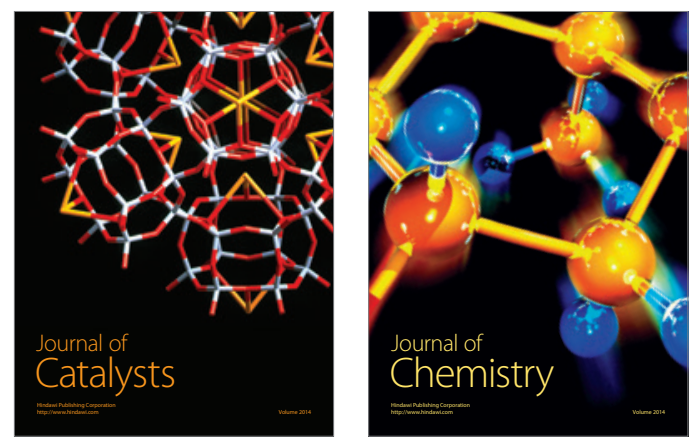
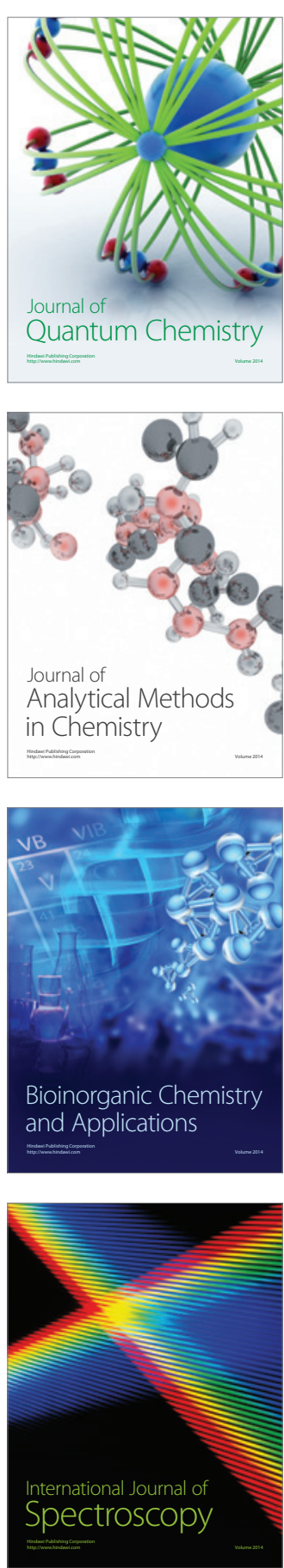